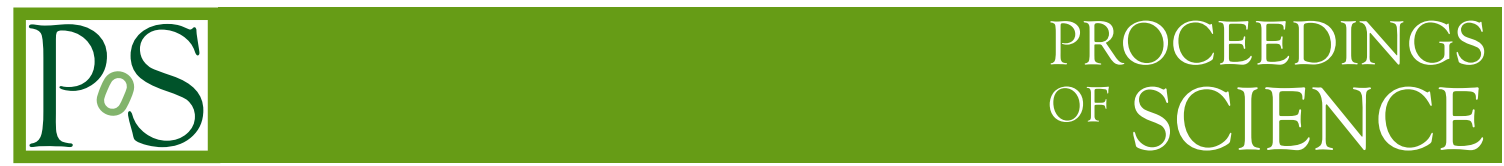

\title{
Polarized Electron and Hadron Beams at eRHIC
}

\section{Christoph Montag*t}

Brookhaven National Laboratory, Upton, NY 11973, U.S.A.

E-mail: montag@bnl.gov

The electron ion collider (EIC) eRHIC at BNL aims at a luminosity of $10^{34} \mathrm{~cm}^{-2} \mathrm{sec}^{-1}$ in collisions of polarized electron and polarized proton, deuteron, and ${ }^{3} \mathrm{He}$ beams. We will present an overview of the proposed facility with an emphasis on generation and acceleration of the polarized beams and the expected polarization performance.

The 18th International Workshop on Polarized Sources, Targets, and Polarimetry, PSTP2019

23-27 September, 2019

Knoxville, Tennessee

* Speaker.

${ }^{\dagger}$ Work supported by Brookhaven Science Associates, LLC under Contract No. DE-AC02-98CH10886 with the U.S. Department of Energy. 


\section{Introduction}

The electron ion collider will answer fundamental questions in nuclear physics and quantum chromodynamics, like the origin of the spin and mass of the proton. The U.S. nuclear physics community, with worldwide support, has compiled a detailed description of the physics program of such a machine and listed the requirements of the future electron ion collider in a White Paper [1]:

- High electron proton luminosity in the range of $10^{33}$ to $10^{34} \mathrm{~cm}^{-2} \mathrm{sec}^{-1}$;

- Large center-of-mass energy range from 20 to $100 \mathrm{GeV}$, upgradable to $140 \mathrm{GeV}$;

- Polarized electron and light ion $\left(\mathrm{p}, \mathrm{d},{ }^{3} \mathrm{He}\right)$ beams with flexible spin patterns;

- Large range of hadron species, from protons to uranium.

The electron ion collider eRHIC, to be constructed at Brookhaven National Laboratory, meets these requirements, without the need for a future energy upgrade to $140 \mathrm{GeV}$.

The eRHIC design is based on the existing RHIC facility [2], including its hadron injector complex. One of the two superconducting RHIC collider rings will serve as the eRHIC hadron storage ring, while the second ring may be used as a full energy polarized hadron injector. A few modifications are necessary to make the RHIC ring suitable as the eRHIC hadron ring, among them removal of the DX separator dipoles in the existing interaction regions which presently limit the beam energy. Thanks to its large circumference of $3.8 \mathrm{~km}$ the hadron ring will reach a proton energy of $275 \mathrm{GeV}$ in this configuration.

An electron storage ring with its injector complex will be installed in the RHIC tunnel, as schematically shown in Figure 1. This ring will be capable of storing electron beams with intensities up to $2.5 \mathrm{~A}$ and energy up to $18 \mathrm{GeV}$. The stored beams will be brought into collision in up to two interaction regions. Based on the needs of the physics program, these interaction regions can be optimized for different center-of-mass energies. Figure 2 shows two sample luminosity curves, optimized at different energies, together with a schematic depiction of the energy and luminosity needs of different physics programs.

\section{Electron Polarization}

Electron beams in storage rings generally self-polarize due to the Sokolov-Ternov effect [3]. In an ideal machine without any depolarizing mechanisms, this results in a maximum polarization level of 92.4 percent. In the eRHIC electron storage ring, the self-polarization time constant ranges from $30 \mathrm{~min}$ to several hours, depending on beam energy. While the eRHIC physics program requires arbitrary spin patterns in both the electron and the proton beam, self-polarization would result in all bunches being polarized anti-parallel to the main dipole field. Self-polarization is therefore not suitable for eRHIC.

To store bunches with arbitrary spin patterns in the electron storage ring requires a full energy polarized injector that injects polarized electron bunches into the storage ring at collision energy with the desired spin orientation. Due to the Sokolov-Ternov effect, bunches with their 


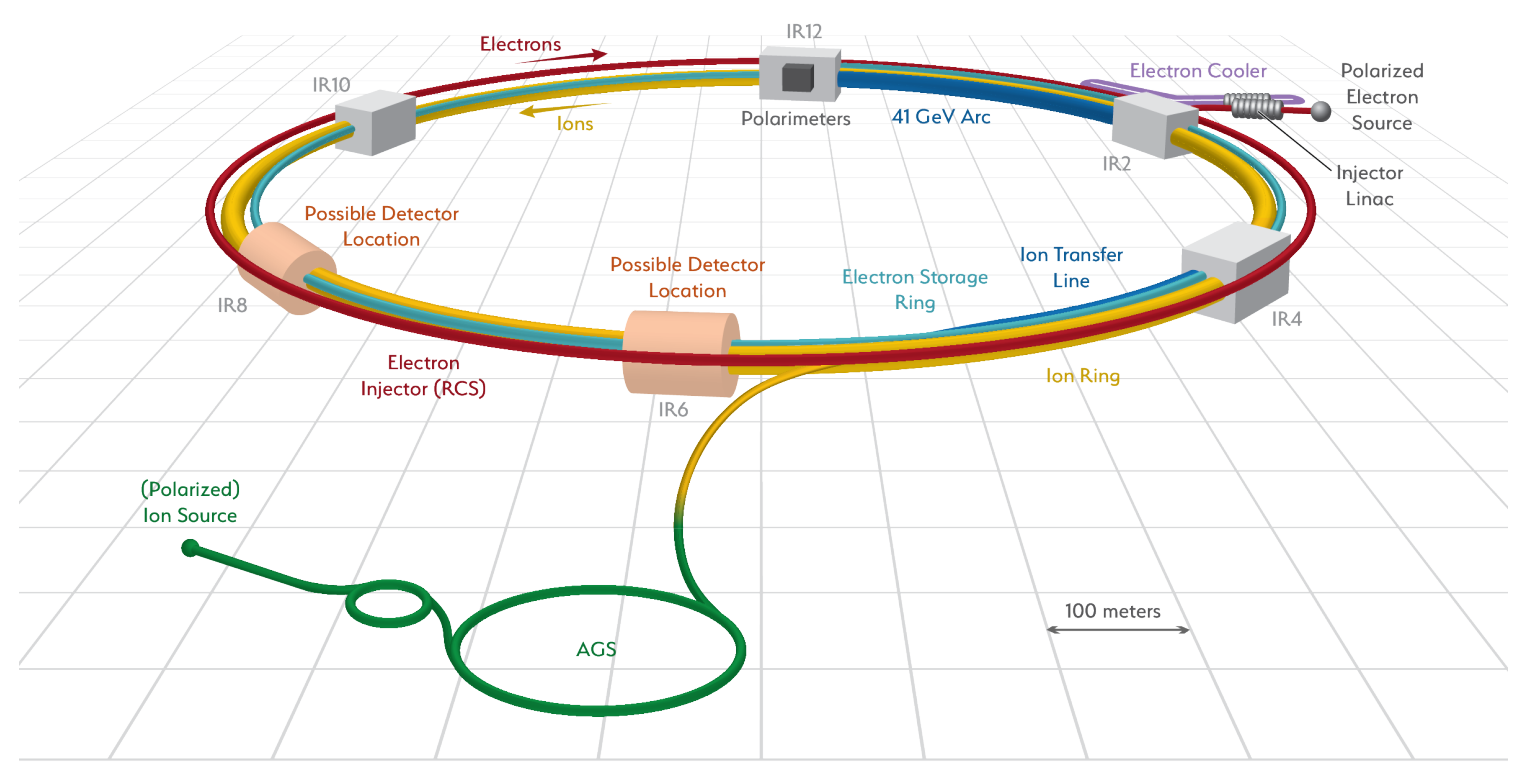

Figure 1: Schematic layout of the eRHIC complex in the existing RHIC tunnel.

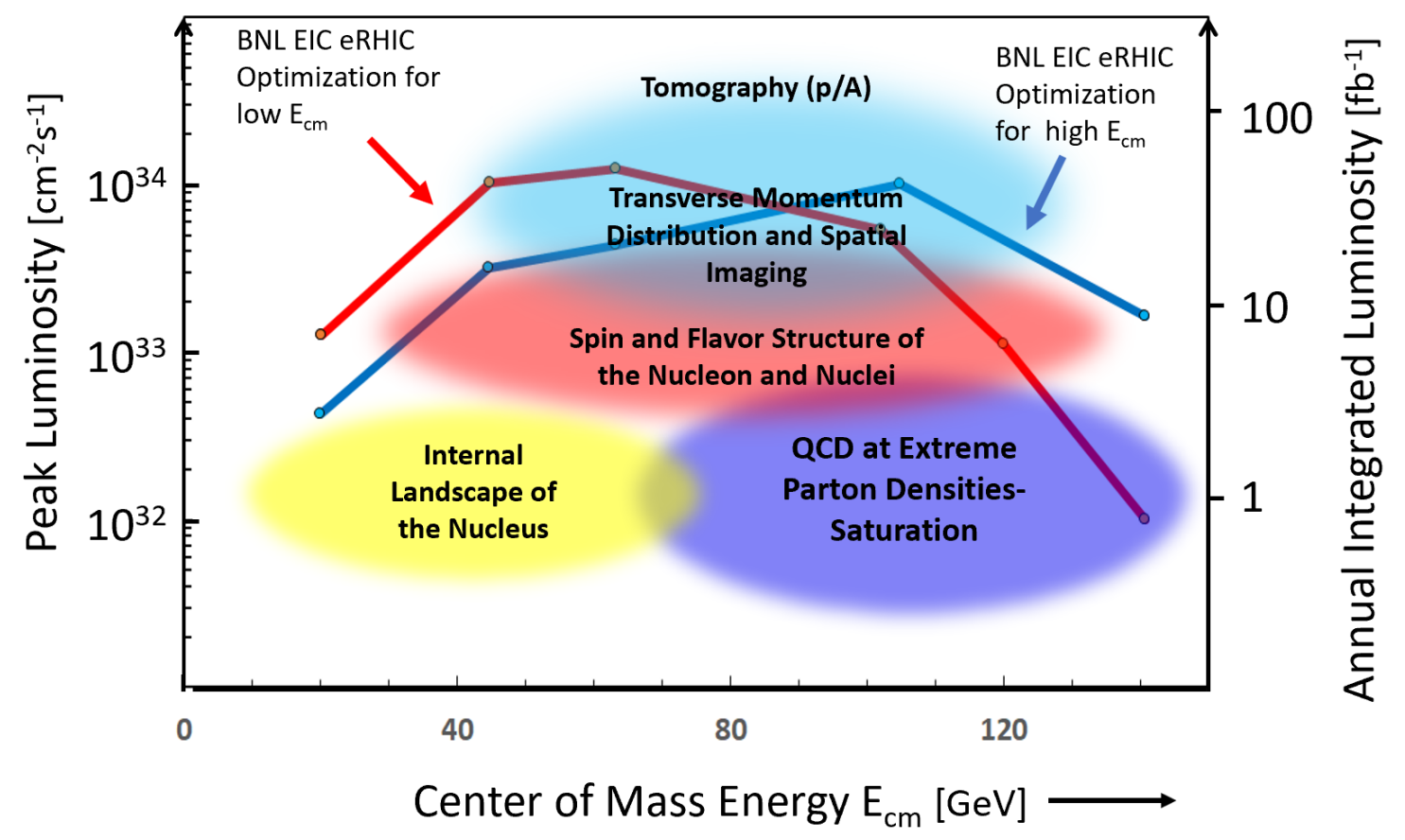

Figure 2: Sample of two luminosity curves optimized at different center-of-mass energies, $63 \mathrm{GeV}$ (red) and $105 \mathrm{GeV}$ (blue), to meet different physics needs. Either optimization yields $10^{34} \mathrm{~cm}^{-2} \mathrm{sec}^{-1}$ luminosity. 
spins aligned parallel to the main dipole field will then begin to first depolarize and eventually selfpolarize in the direction anti-parallel to the dipole field. Since the time constant for this process is much shorter than the desired length of the physics store, the actual storage time for each individual bunch has to be kept short such that only a few percent depolarization occurs during that store time. In addition, spin diffusion [5] leads to depolarization of both spin orientations. To maximize the time-averaged polarization, spin diffusion effects have to be minimized by proper spin matching [6], and entire bunches have to be replaced every few minutes.

Assuming 85 percent polarization upon injection into the storage ring, and an equilibrium polarization of 30 percent as a result of Sokolov-Ternov self-polarization and depolarization due to spin diffusion, a time-averaged polarization of 80 percent is achieved by replacing every bunch with its polarization oriented parallel to the main dipole field after 1.2 minutes, and every bunch with its spins anti-parallel to the main dipole field every 3.2 minutes, as illustrated in Figure 3. This is accomplished by a cycling rate of $2 \mathrm{~Hz}$ in the full energy polarized electron injector.

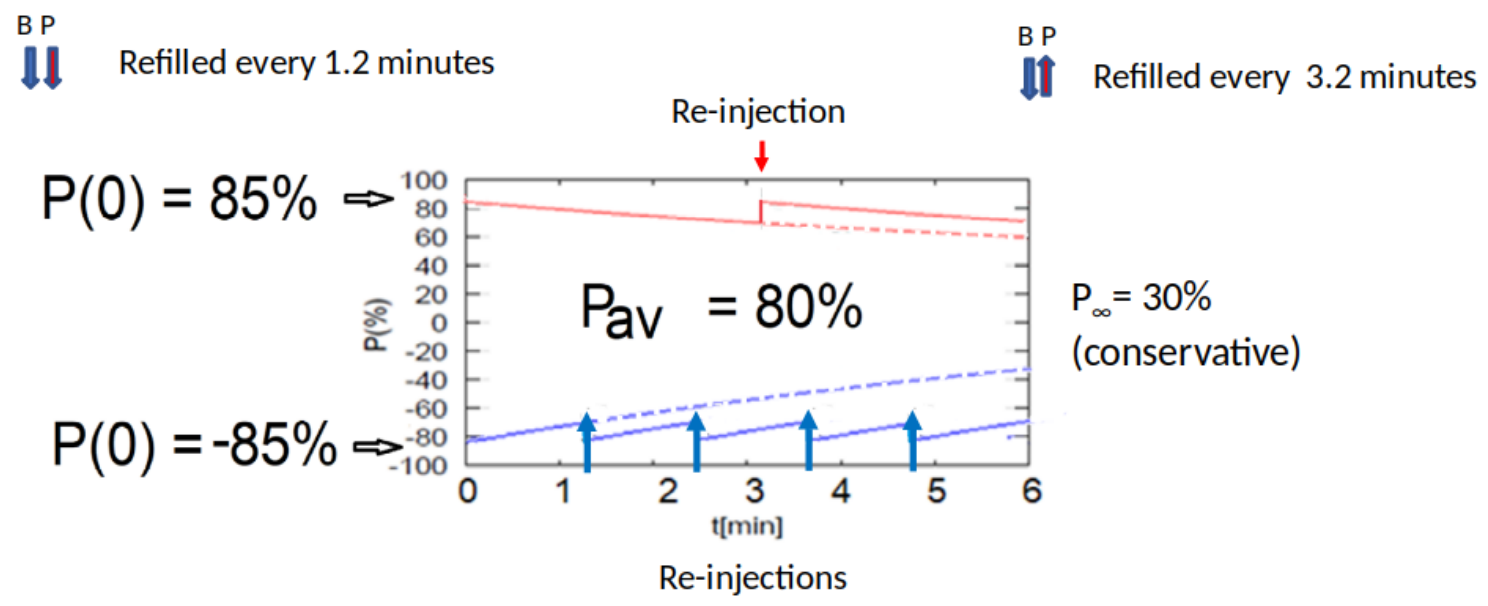

Figure 3: Electron bunch replacement scheme resulting in 80 percent time-averaged polarization [4].

The full energy polarized electron injector is realized as a rapid-cycling synchrotron (RCS). While spin polarization is generally not preserved during the energy ramp in a synchrotron due to the crossing of a large number of depolarizing intrinsic resonances, the dedicatedly spin-resonance free design of this RCS allows for acceleration of polarized electrons to the full energy of $18 \mathrm{GeV}$ [7].

The resonance condition for intrinsic depolarizing resonances can be expressed as

$$
a \gamma=n P \pm Q_{y}
$$

where $a$ is the anomalous gyromagnetic factor of the electron, $\gamma$ the relativistic Lorentz factor, $P$ the periodicity of the synchrotron, and $Q_{y}$ its vertical tune, while $n$ is an integer. The first two intrinsic spin resonances an electron will encounter during acceleration will therefore occur at $a \gamma=Q_{y}$ and at $a \gamma=P-Q_{y}$, assuming $P>Q_{y}$. Thanks to the large circumference of the RCS, $C=3.8 \mathrm{~km}$, a large periodicity of $P=96$ can be realized. Together with its energy range from $400 \mathrm{MeV}$ at injection to $18 \mathrm{GeV}$ at its maximum, $a \gamma$ ranges from 0.9 to 40.8 . Choosing the integer part of the vertical tune as $\left[Q_{y}\right]=50$, these two resonances will therefore be encountered near $a \gamma=50$ and 
$a \gamma=96-50=46$. However, since the maximum value of $a \gamma$ in the eRHIC RCS is only 40.8, these depolarizing resonances are outside the energy range of the machine and will therefore never be encountered. As a side benefit, the imperfection resonances are also minimized due to the design of this lattice.

Extensive simulation studies have been performed to validate this concept. These studies show that the polarization loss during the ramp can be kept below $1 \%$ provided the orbit distortions caused by quadrupole misalignment are corrected to less than $0.5 \mathrm{~mm}$ RMS, and the ramp time is $100 \mathrm{msec}$ or faster, as shown in Figure 4. This required orbit stability is being routinely achieved at the NSLS-II booster [8].

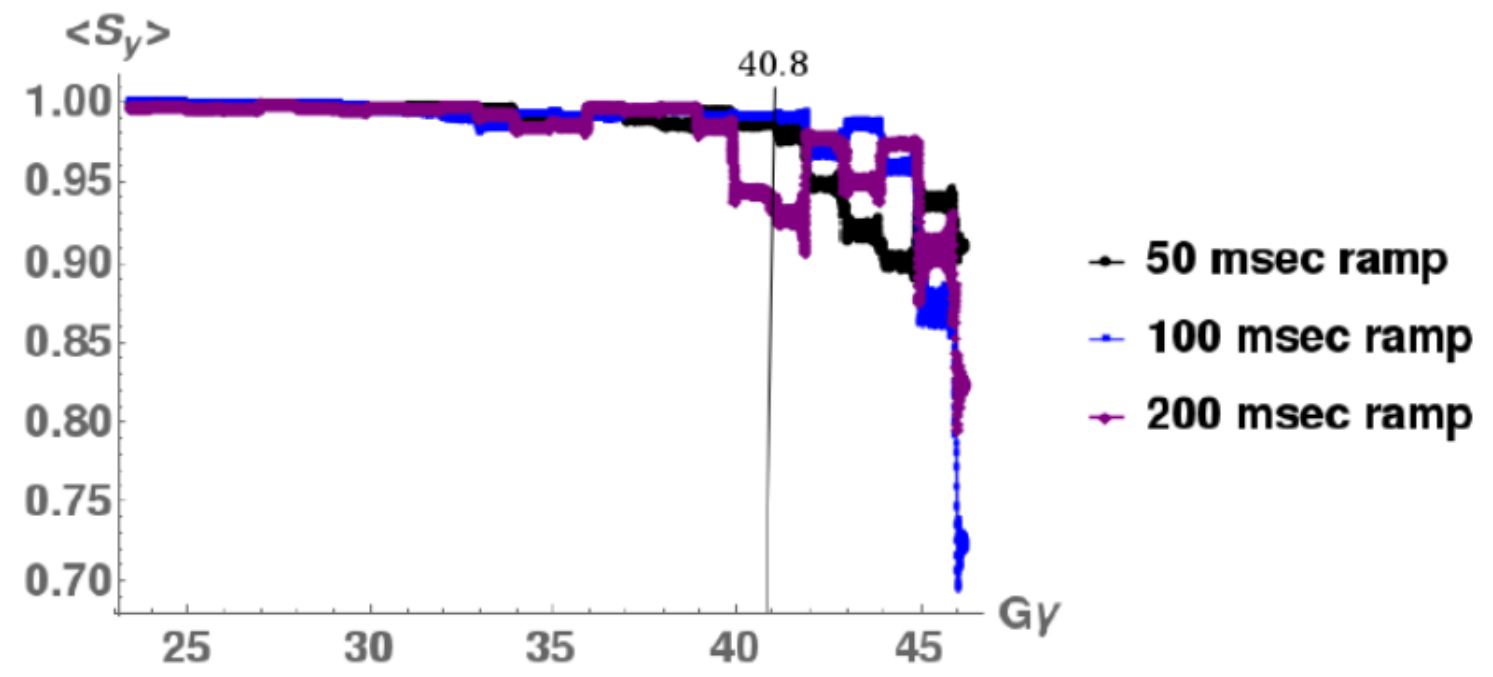

Figure 4: Simulated electron polarization in the RCS during the energy ramp, for three different ramp durations of 50, 100, and $200 \mathrm{msec}$ [4].

\section{Hadron Polarization}

The eRHIC hadron ring will be based on the existing "Yellow" RHIC ring. RHIC has routinely achieved proton polarization levels around 60 percent at $255 \mathrm{GeV}$ beam energy. For eRHIC, this level needs to reach 80 percent.

Presently, the optically pumped polarized ion source (OPPIS) at Brookhaven National Laboratory delivers proton beams with 83 percent polarization. During acceleration in the Booster and the AGS some depolarization occurs, such that the polarization at AGS extraction is reduced to 70 percent. After acceleration to $255 \mathrm{GeV}$ in RHIC with its two Siberian snakes per ring, this value has further decreased to typically 60 percent. Increasing the polarization in the eRHIC hadron ring therefore requires improvements in both the AGS and the hadron ring itself.

The AGS will be equipped with a stronger Siberian snake and skew sextupoles. Together with an increased injection energy a polarization level of 80 percent is expected at extraction into RHIC, based on simulation studies that have been benchmarked with the present AGS configuration. In the eRHIC hadron storage ring, four additional Siberian snakes will be installed, for a total of six. Two of those new snakes will be taken from the "Blue" RHIC ring, while the other two will be assembled 
from the helical dipoles of the spin rotators in the "Blue" ring. Benchmarked simulations show that in this configuration polarization will be completely preserved during the ramp. Thus, 80 percent proton polarization is expected in the eRHIC hadron storage ring.

Besides polarized protons, the eRHIC physics program also requires polarized deuterons as well as polarized ${ }^{3} \mathrm{He}$ ions. Due to their tiny anomalous gyromagnetic factor polarized deuterons encounter only a small number of intrinsic spin resonances during the energy ramp, which can be overcome by implementing a tune jump system. With six Siberian snakes full polarization preservation is expected on the ramp for both deuterons and ${ }^{3} \mathrm{He}$ beams.

\section{Spin Rotators}

While the polarization vectors are oriented parallel to the vertical main dipole field in the arcs, the physics program requires longitudinal polarization at the interaction point (IP). This conversion from vertical to longitudinal orientation is accomplished by pairs of spin rotators in the interaction region straight section.

In the hadron storage ring, the existing spin rotators, based on superconducting helical dipoles [9], will be used. The spin rotators in the electron storage ring will be based on superconducting solenoid magnets. These solenoids rotate the vertical spins from the arc into the radial direction. A subsequent dipole then transforms them from radial to longitudinal orientation. After passing the IP and colliding with the hadron beam, this process is reversed. Each solenoid is split into two segments, with a set of quadrupoles in-between for local coupling compensation.

\section{Summary}

The electron ion collider eRHIC at Brookhaven National Laboratory will collide highly polarized electron and light ion (proton, deuteron, and ${ }^{3} \mathrm{He}$ ) beams, as well as polarized electrons with unpolarized heavy ions up to uranium. The peak electron proton luminosity reaches 1.05 . $10^{34} \mathrm{~cm}^{-2} \mathrm{sec}^{-1}$ at $105 \mathrm{GeV}$ center-of-mass energy. An alternative optimization of the interaction region design and the machine parameters for low energy operations yields a peak luminosity of $1.2 \cdot 10^{34} \mathrm{~cm}^{-2} \mathrm{sec}^{-1}$.

Arbitrary spin patterns ("up" and "down") in both beams will be provided by the respective injectors. While the hadron complex will take full advantage of the existing RHIC injector complex, a spin resonance free rapid cycling synchrotron (RCS) will serve as full energy polarized electron injector into the storage ring. Additional Siberian snakes in the hadron ring will increase its spin preservation on the energy ramp to nearly 100 percent.

\section{References}

[1] A. Accardi et al., "Electron Ion Collider: The Next QCD Frontier", Eur. Phys. J., vol. A52, no. 9, p. 268, 2016.

[2] M. Harrison, T. Ludlam, and S. Ozaki, "RHIC Project Overview", Nucl. Instr. Meth. A, vol. 499 (2-3), p. 235, 2003. 
[3] A. Sokolov and I. Ternov, "On polarization and spin effects in the theory of synchrotron radiation" (In English, Russian), Sov. Phys. Dokl. 8 (1964) no. 12, p. 1203-1205, Dokl. Akad. Nauk SSSR 153 (1963) no. 5, p. 1052-1054.

[4] A. Arno et al., "Electron Ion Collider eRHIC at Brookhaven National Laboratory - Pre-Conceptal Design Report 2019”, BNL Formal Report BNL-211943-2019-FORE, 2019.

[5] Y. Derbenev and A. Kondratenko, "Diffusion of particle spins in storage elements", Sov. Phys. JETP, vol. 35, p. 230, 1972.

[6] V. Ptitsyn, Y. Shatunov, and S. Mane, "Spin response formalism in circular accelerators", Nucl. Instr. Meth. A, vol. 608, p. 225, 2009.

[7] V. Ranjbar et al., "Spin resonance free electron ring injector", Phys. Rev. Accel. Beams 21 (2018) no. $11,111003$.

[8] Guimei Wang, private communication.

[9] V. Ptitsin and Y. Shatunov, "Helical spin rotators and snakes", Nucl. Instr. Meth. A, vol. 398, p. 126, 1997. 